\title{
Low-Cost QCM Sensor System for Screening Semen Samples
}

\author{
Michael I. Newton, Shaun Atherton, Robert H. Morris, Simon M. Stanley, \\ Carl R. Evans, David C. Hughes, and Glen McHale
}

School of Science and Technology, Nottingham Trent University, Clifton Lane, Nottingham NG11 8NS, UK

Correspondence should be addressed to Michael I. Newton, michael.newton@ntu.ac.uk

Received 8 September 2010; Revised 28 October 2010; Accepted 23 November 2010

Academic Editor: S. C. Mukhopadhyay

Copyright (C) 2010 Michael I. Newton et al. This is an open access article distributed under the Creative Commons Attribution License, which permits unrestricted use, distribution, and reproduction in any medium, provided the original work is properly cited.

Artificial insemination is a well-established part of modern agricultural practice. A viable semen sample is judged by the total number of spermatozoa (sperm) in the sample and the motility of the sperm. In this paper, we report the development of a reusable measurement cell and electronics for screening semen samples based on the Quartz Crystal Microbalance (QCM) and Universal Frequency to Digital Converter (UFDC-1) to produce a low-cost sensor system. After introducing the semen sample at one end of the measurement cell, sperm swim down a channel before causing a frequency change on the QCM. Data is presented that shows the different frequency changes using a commercial frequency counter caused by porcine semen samples, one two days old and one twenty one days old. Similar data is presented for a motile semen sample measurement using the low-cost UFDC-1.

\section{Introduction}

Artificial insemination of animals is a routine element in modern agricultural practice. For most species, semen can reliably be frozen and stored for long periods of time before being thawed and used. For some species, however, the freezing process is less reliable requiring that the semen be shipped chilled and used within several days. Semen analysis is currently a specialist, subjective, and skilled process that is normally carried out under laboratory conditions. The tests usually look at the number of spermatozoa (sperm) in a given volume of semen and also their ability to swim, that is, their motility. Most samples are analysed using optical techniques such as a haemocytometer or counting chamber [1]. Alternatively, they may rely on expensive computer aided semen analysis [2] or a combination of fluorescent staining and flow cytometry to analyze thousands of sperm in a sample [3]. These laboratory-based techniques are not suitable for use on a farm to test samples immediately prior to insemination. Under such conditions, a robust instrument that gives a simple yes or no answer without requiring complex sample preparation or interpretation of results is required. In a first report in 2007, we suggested that a simple time of flight technique using an acoustic wave sensor could provide a real-time screening technique for monitoring the motility of sperm in a semen sample [4]. The screening process is not designed to give a detailed analysis of a sample but rather determine if there is greater than a threshold number of motile sperm. It is known that the operation of a quartz crystal microbalance (QCM) or thickness shear mode device depends on the density and viscosity of the medium [5] and that sperm represent a complex object which extends beyond the penetration depth of a QCM. To demonstrate that sperm attachment could be treated like a rigid mass, the frequency change of a quartz crystal due to attached sperm was measured, and the number of attached sperm counted under a microscope [4]. It was demonstrated that the attachment of the sperm to the QCM could be modelled using the Sauerbrey equation [6] with an "effective" mass for a single porcine sperm of around $5 \mathrm{pg}$.

The technique was then further developed to consider some of the operating parameters including the effect of temperature and the presence of progesterone in the swim media [7]. In this paper, we report the development of a suitable swim cell and electronic measurement system based on the universal frequency to digital converter (UFDC1, IFAC) that offers a low-cost sensor system suitable for screening a semen sample at the point of use on a farm. 


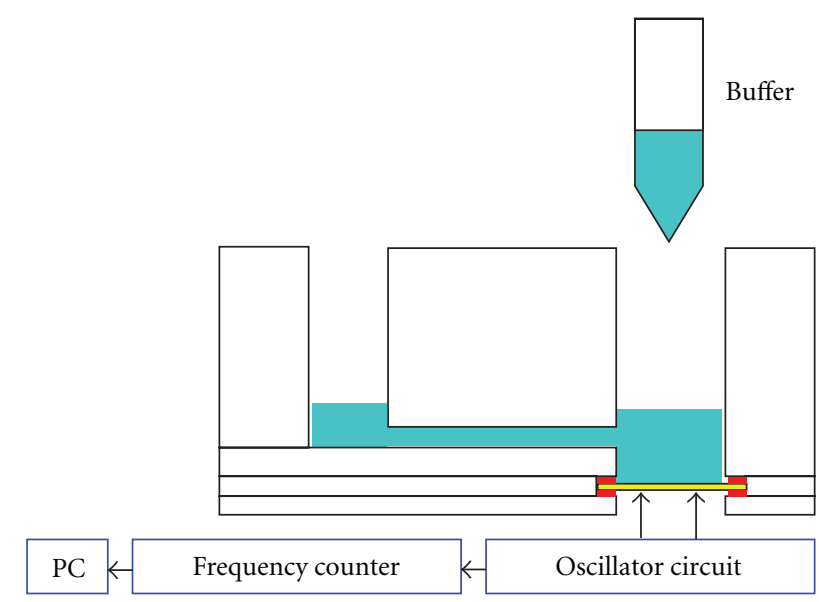

(a)

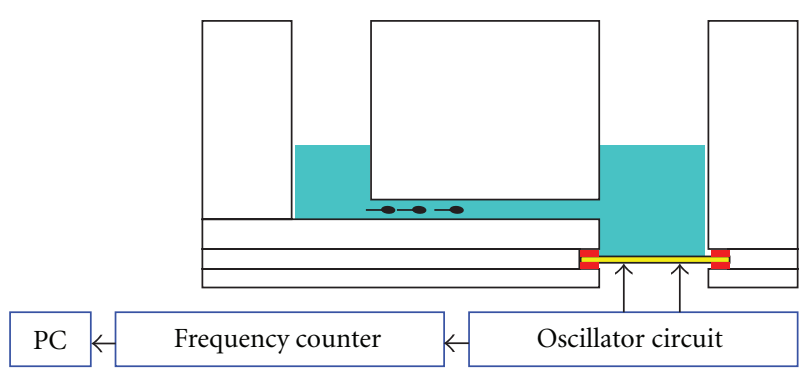

(c)

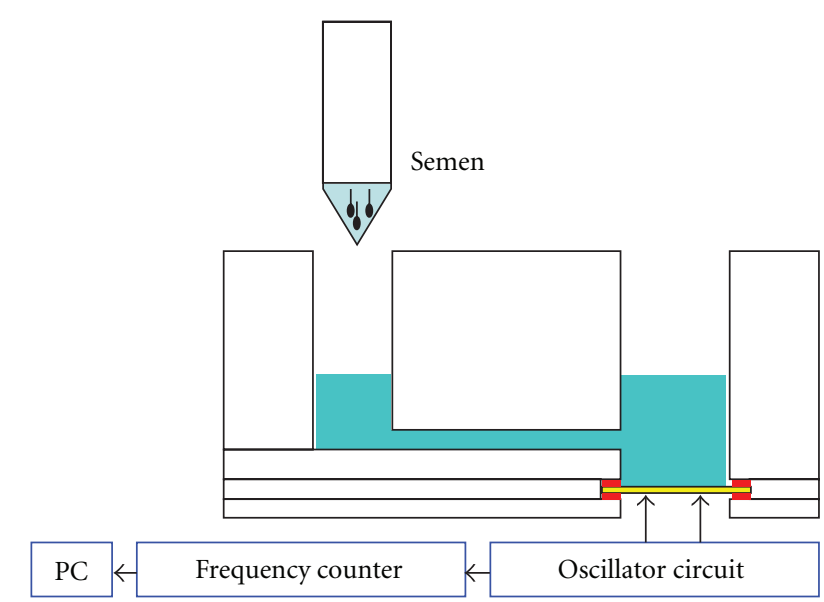

(b)

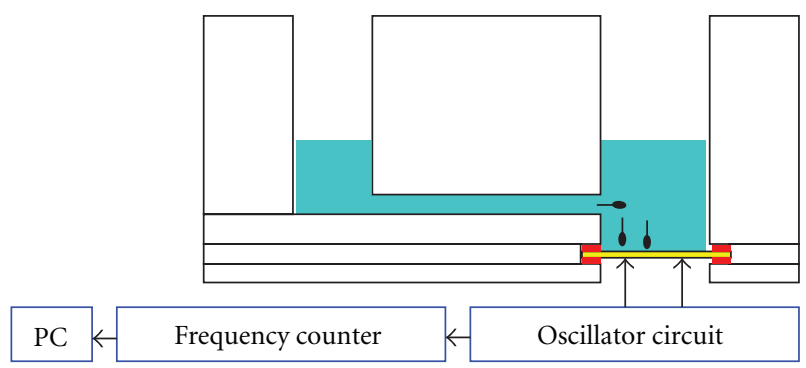

(d)

FIGURE 1: Schematic diagram of the acrylic swim channel with the quartz crystal (yellow) held in place with rubber gasket (red). The measurement sequence starts with buffer introduced into the swim cell (a). The semen sample is introduced at the opposite end of the swim channel to the quartz crystal (b). The sperm swim down the swim channel (c) and attached to the quartz crystal causing a frequency change (d).

\section{Materials and Methods}

Figure 1 shows a schematic diagram and example sequence of a typical experiment. The "swim" cell was produced using acrylic sheet cut on an M-500 laser cutter (Universal Laser Systems) and bonded using Extrufix (Bostik). A $12 \mathrm{~mm}$ diameter AT-cut quartz crystal with wrap around electrodes (Laptech XL1052) and fundamental frequency $10 \mathrm{MHz}$ was clamped into the cell using rubber gaskets. Contact was made to the crystal using spring-loaded contacts directly connected to the oscillator circuit described below. The swim cell provided an opening for introducing the $1.5 \mathrm{~mL}$ of phosphate buffered saline (PBS) swim media (Figure 1(a)) and a smaller opening at the end of a $15 \mathrm{~mm}$ long swim channel for introduction of the semen sample (Figure 1(b)). After the semen is introduced, the sperm swim down the channel (Figure 1(c)) attaching to the quartz crystal at the end (Figure 1(d)) causing a change in the resonant frequency. For the initial development work, a universal frequency counter (Agilent 53132A) interfaced to a computer was used to measure the frequency every half a second. The instrument is intended as a screening device rather than an analytical device so it is only required to test against a threshold frequency change.
The porcine semen was supplied by a commercial artificial insemination centre (JSR Genetics, Driffield, UK). Commercial suppliers usually provide the semen in buffer and include other chemicals to prolong semen life. This allows the semen to be stored unopened for up to 5 days below $17^{\circ} \mathrm{C}$ without extensive deterioration in semen motility. To attain a sample of immotile sperm for comparison, we stored an unopened sample for 21 days prior to use. In our previous reports $[4,7]$, we investigated coatings of cysteamine and Poly-L-Lysine on the quartz crystal that would act as an adhesive layer for the sperm. In this work, we found that a clean gold electrode on a polished quartz crystal was as efficient and more reliable than the chemical modifications previously tested; this could then be cleaned for repeated usage using a Papain solution (Sigma Aldrich P3375-25G) or physical agitation with a cotton bud and adequate rinsing with PBS solution.

The design of a low-cost high-stability quartz oscillator for operation in liquids is challenging and has recently been reviewed by Arnau [8]. In this work, we have produced a simple circuit based on the Pierce oscillator using a MAN1LN amplifier (Mini-Circuits). The MAN-1LN is a lownoise amplifier operating from $0.5 \mathrm{MHz}$ to $500 \mathrm{MHz}$ with a gain of around $28 \mathrm{~dB}$ operating from a nominal $12 \mathrm{~V}$ 


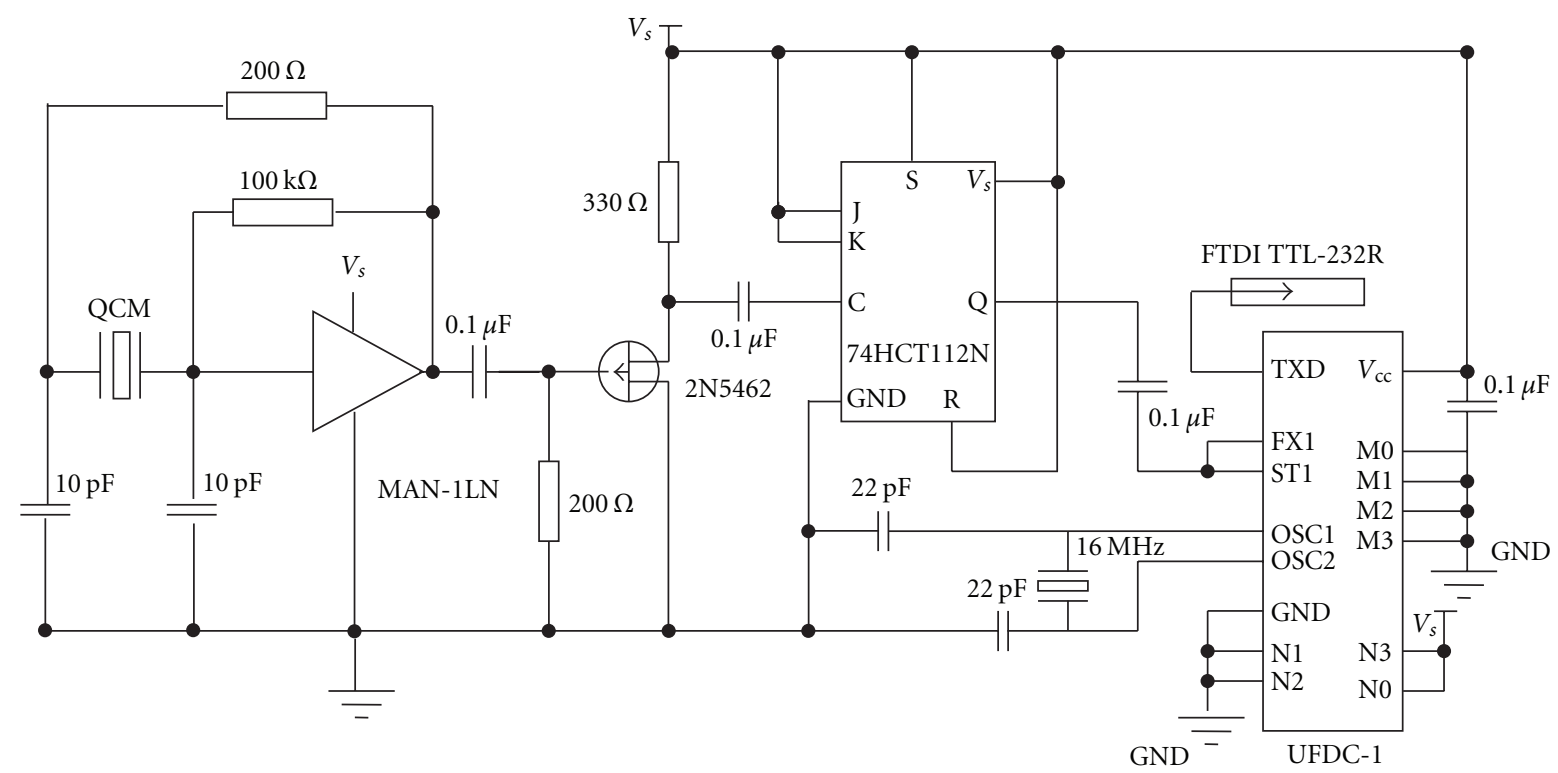

FIGURE 2: Schematic diagram of the oscillator and frequency counter electronics.

supply. This oscillator offers good long-term stability giving a frequency drift of around $2 \mathrm{~Hz}$ in an hour in the air. In PBS, the two-sample Allan variance for the oscillator has been calculated to be $3 \times 10^{-7}$ which is comparable to the results reported for a $2 \mathrm{MHz}$ oscillator in water by RodriguezPardo et al. [9] although our value is greater than they report for a $9 \mathrm{MHz}$ QCM. A UFDC-1 universal frequency to digital converter [10] was used as a low-cost replacement for the Agilent frequency counter. The UFDC- 1 is a 28 pin single-chip programmable two-channel frequency-todigital converter, operating over the frequency range $0.05 \mathrm{~Hz}$ to $7.5 \mathrm{MHz}$; this range can be extended to $120 \mathrm{MHz}$ with prescaling although this results in an accuracy reduction. The conversion accuracy is programmable from $1 \%$ to $0.001 \%$ and is determined by selecting which of the four pins on the UFDC-1 labelled N0-N3 are connected to either the supply voltage or ground: the mode of operation is selected in a similar way using pins M0-M3. Data is returned as a string of ASCII characters (note that the number of decimal places shown by this string is not the same as the actual accuracy of the conversion) from the serial output pin (TXD). The serial output is ideal for use with a USB to TTL serial cable (FTDI TTL-232R-3V3). The UFDC-1 operates from a single power supply of between $+4.5 \mathrm{~V}$ and $+5.5 \mathrm{~V}$ which is taken from the USB port. The FTDI drivers enable the USB to be accessed as a standard COM port. A program was written in the free programming language Just Basic (http://www.justbasic.com/) to read the data from the COM port, extract the string for a single value, convert to a number, and store it to a spreadsheet file. Given the maximum frequency of the UFDC-1 without prescaling is $7.5 \mathrm{MHz}$, operation of the QCM in the "swim cell" at $10 \mathrm{MHz}$ was above this frequency, and requires a divide by two pre-scalar. The thickness of the crystal reduces with increasing frequency and whilst the $10 \mathrm{MHz}$ are sufficiently robust, $15 \mathrm{MHz}$ crystals proved to be too fragile for practical operation in this application. In addition to the oscillator, the circuit diagram in Figure 2 shows the frequency counter and buffer circuit. The sinusoidal output of the oscillator has a peak-to-peak amplitude around $7 \mathrm{~V}$ which is fed into an FET operated in switch mode between $0 \mathrm{~V}$ and $+5 \mathrm{~V}$ from the USB. The digital signal is then fed into a divide by two circuit consisting of a single flip flop on a $74 \mathrm{HCT} 112 \mathrm{~N}$ and then into the F1 input of the UFDC-1 operating in mode zero (singlechannel frequency measurement); for improved stability, the analogue part of the circuit (oscillator) was operated from a dc battery supply and LM3171 voltage regulator and the digital part of the circuit supplied from the $+5 \mathrm{~V}$ of the USB. Figure 3(a) shows a photograph of the swim cell with the box below including the oscillator circuit, and Figure 3(b) shows a photograph of the circuit board for the UFDC-1 frequency counter circuit.

\section{Results and Discussion}

Whilst initial stability tests in the air were good, when the cell contained only PBS, it exhibited periodic oscillations in the frequency of several hundred $\mathrm{Hz}$. In the experiments with water, the change in depth required to move through one pressure wave resonance cycle is only about $70 \mu \mathrm{m}[11]$, and in an open cell of the type we are using, this can occur by evaporation of the PBS in a relatively short time. As a result of this, we used a simple flat sheet of pressure sensitive adhesive (Blu-tac, Bostik) inserted into the PBS above the QCM at an angle of 45 degrees, and this reduced the frequency drift on the bench top in an open lab to around $10 \mathrm{~Hz}$ in 40 minutes.

Semen samples were introduced using a pipette and vigorous use could result in the sample attaining sufficient momentum so as not to reflect the actual motility of the sperm. Initial tests with the aged semen did show a significant 


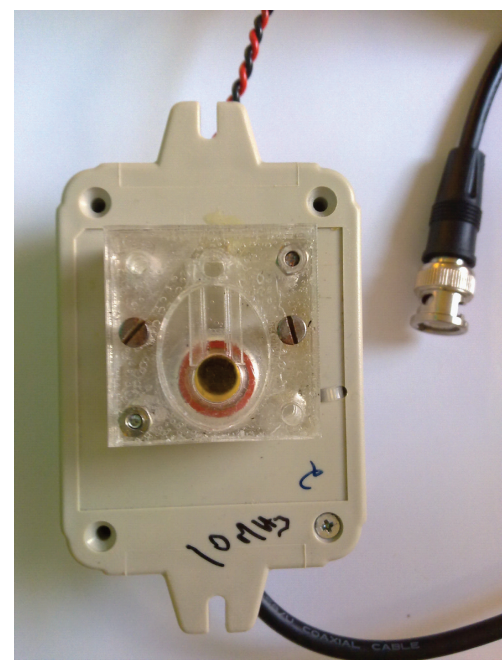

(a)

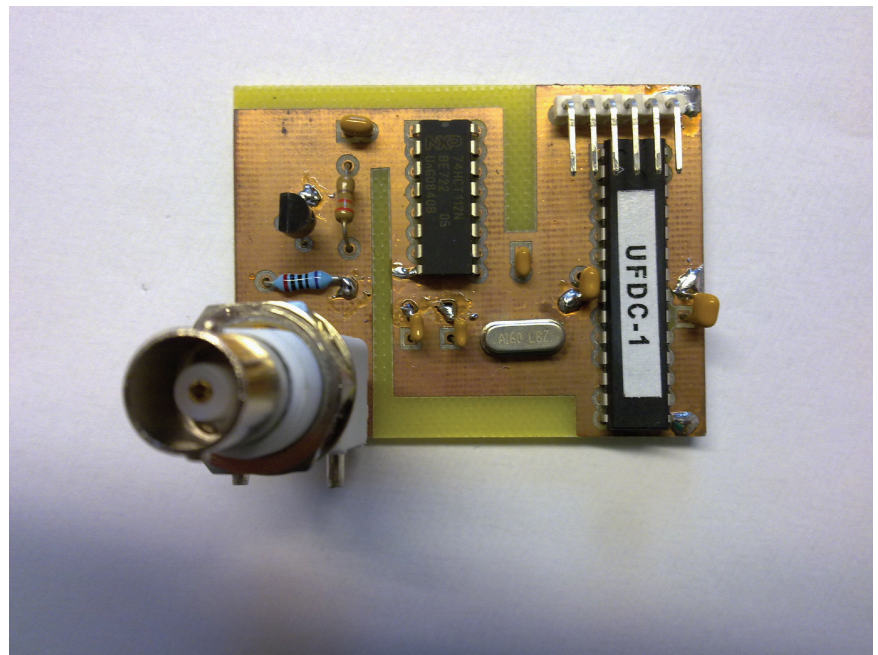

(b)

FIGURE 3: (a) Image of quartz crystal in acrylic cell with the oscillator circuit in the box under the cell. (b) Image of UFDC-1 circuit.

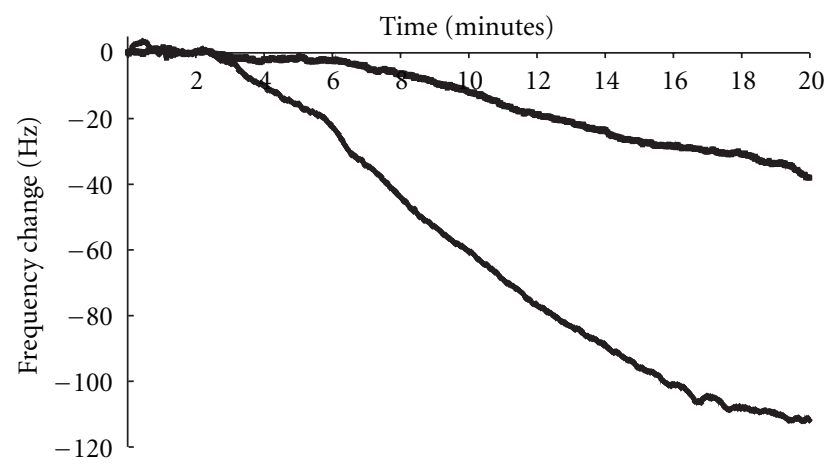

Figure 4: Frequency change as a function of time for twenty one day-old sample (upper line) and two-day-old sample (lower line).

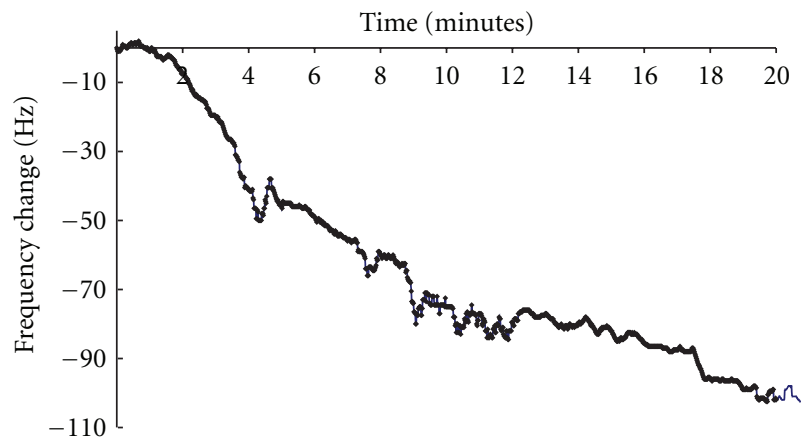

FIGURE 5: Frequency change as a function of time for the UFDC-1 from introduction $50 \mu \mathrm{L}$ of four-day-old semen sample.

effect which could be comparable in frequency shift to "live" semen that was several days old. A simple tilting of the whole swim cell by ten degrees reduced the effect of this to a manageable level by effectively providing a small well. Figure 4 shows the data using the Agilent frequency counter for $10 \mu \mathrm{L}$ of 2-day-old semen sample and a 21-dayold semen sample of the same volume. Although a change of $35 \mathrm{~Hz}$ is observed over twenty minutes, this is sufficiently low to discriminate a viable from nonviable semen sample for a simple screening application and probably represents simple diffusion. Remembering that screening just requires a simple "yes" or "no" if the sample is viable or not, after a given measurement time of say 10 minutes, the "yes" answer would be given if the frequency change had exceeded $50 \mathrm{~Hz}$; otherwise, the "no" would be given. Most of the semen in the older sample was still visibly collected in the well whilst for the 2-day-old sample significant observed at the QCM. Sufficient data is collected to allow checks that the system is working correctly as, a frequency change of greater than say $1 \mathrm{kHz}$ would represent a fault having occurred and render the current measurement invalid. In Figure 5, we show the frequency change as a function of time for the UFDC-1 from introduction of $50 \mu \mathrm{L}$ of four-day-old semen. A moving average has been applied to the UFDC data which has a frequency resolution of $5 \mathrm{~Hz}$; data was collected from the UFDC-1 every second. Whilst a slightly lower overall frequency change is observed than shown by the Agilent frequency counter in Figure 4, this is consistent with the aging of the semen sample.

\section{Conclusions}

The reusable sensor system we have reported here is designed for simple screening for sufficiently motile sperm in a sample; that is, that a threshold frequency change has been exceeded. As commercial semen samples are significantly diluted with an extender to maintain the quality during transport, this threshold will depend on the particular application. The oscillator circuit presented is simple, inexpensive, and sufficiently stable for such an application. The frequency counter comprised of the UFDC- 1 and TTL-USB cable, 
forms a complete low-cost and light-weight system such that the components of the circuit shown in Figure 3(b) with the cable is under US\$50; this may provide a suitable platform for other QCM-based applications. An improved "swim cell" design will incorporate the angle and the baffle to prevent resonance conditions being encountered. This system should allow for a great improvement in the reliability and ease of use of onsite field measurements of sperm motility.

\section{Acknowledgments}

This work has been supported by the UK Engineering and Physical Sciences Research Council (EPSRC) under Grant no. EP/E063489/1 and Higher Education Innovation and Regional Fellowship (HIRF409).

\section{References}

[1] World Health Organization, WHO Laboratory Manual for the Examination of Human Semen and Sperm-Cervical Mucus Interaction, Cambridge University Press, Cambridge, UK, 3rd edition, 1999.

[2] S. T. Mortimer, "CASA - practical aspects," Journal of Andrology, vol. 21, no. 4, pp. 515-524, 2000.

[3] P. Christensen, D. Boelling, K. M. Pedersen, I. R. Korsgaard, and J. Jensen, "Relationship between sperm viability as determined by flow cytometry and nonreturn rate of dairy bulls," Journal of Andrology, vol. 26, no. 1, pp. 98-106, 2005.

[4] M. I. Newton, C. R. Evans, J. J. Simons, and D. C. Hughes, "Semen quality detection using time of flight and acoustic wave sensors," Applied Physics Letters, vol. 90, no. 15, Article ID 154103, 2007.

[5] K. K. Kanazawa and J. G. Gordon, "The oscillation frequency of a quartz resonator in contact with liquid," Analytica Chimica Acta, vol. 175, no. C, pp. 99-105, 1985.

[6] G. Sauerbrey, "The use of quartz oscillators for weighing thin layers and for microweighing," Zeitschrift Fur Physik, vol. 155, pp. 206-222, 1959.

[7] S. Atherton, C. R. Evans, P. Roach, D. C. Hughes, G. McHale, and M. I. Newton, "Investigation of operating parameters for a semen quality analysis system," in Proceedings of the 2nd International Conference on Biomedical Electronics and Devices (BIODEVICES '09), pp. 13-16, January 2009.

[8] A. Arnau, "A review of interface electronic systems for AT-cut quartz crystal microbalance applications in liquids," Sensors, vol. 8, no. 1, pp. 370-411, 2008.

[9] L. Rodriguez-Pardo, J. Fariña, C. Gabrielli, H. Perrot, and R. Brendel, "Resolution in quartz crystal oscillator circuits for high sensitivity microbalance sensors in damping media," Sensors and Actuators B, vol. 103, no. 1-2, pp. 318-324, 2004.

[10] S. Y. Yurish, "Digital sensors design based on universal frequency sensors interfacing IC," Sensors and Actuators A, vol. 132, no. 1, pp. 265-270, 2006.

[11] S. M. Reddy, J. P. Jones, and T. J. Lewis, "Use of combined shear and pressure acoustic waves to study interfacial and bulk viscoelastic effects in aqueous polymeric gels and the influence of electrode potentials," Faraday Discussions, vol. 107, pp. 177196, 1997. 

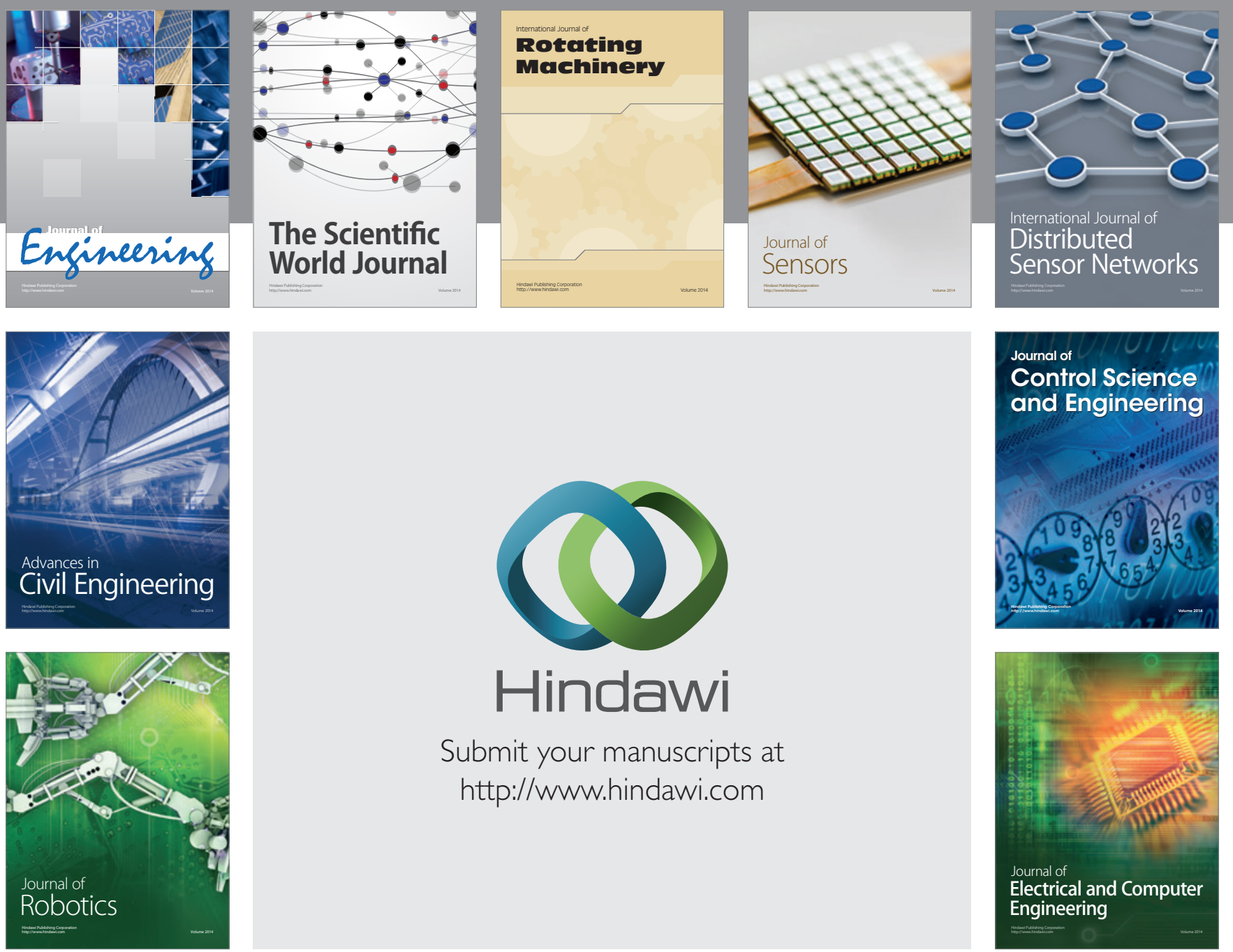

Submit your manuscripts at

http://www.hindawi.com
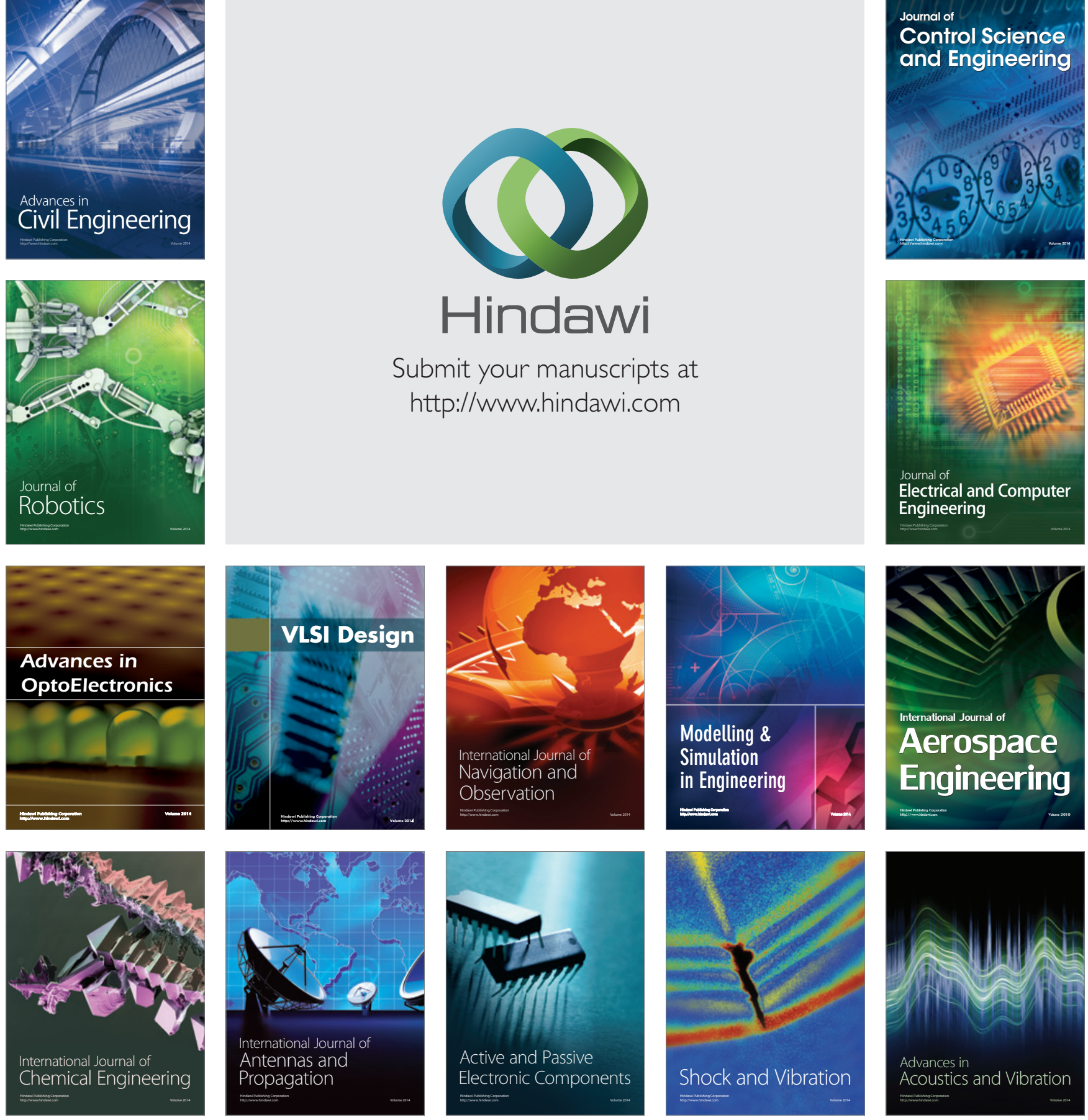